\title{
Linear Stability of Flows in a Squeeze Film *
}

\author{
ZHU Ke-Qin (朱克勤) $)^{* *}$, REN Ling(任玲 $)^{1}$, LIU Yi(刘祎) $)^{2}$ \\ ${ }^{1}$ Department of Engineering Mechanics, Tsinghua University, Beijing 100084 \\ ${ }^{2}$ Department of Mechanical Engineering, California Institute of Technology, CA, 91125, USA
}

(Received 3 March 2005)

\begin{abstract}
We study linear stability of viscous flows in a squeeze lubrication film, in which the flow varies slowly in space and time, between two parallel plates moving normal to each other with a slow constant speed, generalizing the inviscid results of Aristov and Gitman [J. Fluid Mech. 464 (2002) 209]. The temporal evolution of twodimensional disturbances for this physical situation, including the asymptotic behaviour of a long term or the transient behaviour of some time interval, is obtained by the construction of a low-dimensional Galerkin method. It is found that the wall boundaries typically play dual roles of stabilizer and destabilizer. They constrain the development of disturbances and have stabilizing influences. However, they give rise to velocity shear, which is diffused by viscosity and thereby tends to destabilize the flow.
\end{abstract}

PACS: 47.15.Fe, 47.20.Gv

It is well known that some processes, which are frequently encountered in industry, such as the motion of a liquid through a hydraulic pump or a damper, can be considered as the flow between two parallel walls which move normal to each other with a constant speed. Assuming the breadth of plates to be much larger than the distance between them, the model is related to the lubrication theory. If the basic flow is disturbed slightly, the growth and decaying of the disturbance relates to the linear stability. It is therefore of great interest and significance to investigate the possible instability in the squeeze flow near the stagnation point.

The concept of the instability for a steady parallel flow, such as the plane Poiseuille flow, ${ }^{[1]}$ is very typical in the hydrodynamic stability theory. Except for steady basic flows, there has been rapidly growing literature on the stability of parallel flows that vary slowly in time, for example the slowly varying timedependent flows between two concentric cylinders. ${ }^{[2]}$ Other than strictly parallel flows, most shear flows of interest, such as jets, wakes and boundary layers under some conditions, are weakly non-parallel flows that change slowly in one space variable. More complicated flows combine the unsteady and weakly non-parallel characteristics: i.e., basic flows are characterized by the slow changes in space and time, such as the flow between parallel walls moving normal to each other with a slow speed, ${ }^{[3-5]}$ as is discussed in the present study.

When two parallel plates slowly move towards each other or in opposite directions, the gap between the plates is dependent on time, and the basic flow depends slowly on time and does not change its magnitude much after a long time. In addition, the horizontal velocity components, $x^{*}$ and vertical $z^{*}$ in a two-

dimensional squeeze-lubrication film satisfy $u_{x}^{*} \gg u_{z}^{*}$ and $\partial u_{x}^{*} / \partial x^{*} \ll 1$, where $u_{x}^{*}$ is a function of coordinates $x^{*}$ and $z^{*}$ and the asterisked variables are dimensional, characterizing the slow change in one space variable. Therefore, the basic flow belongs to a sort of the weakly non-parallel and slowly varying timedependent flow.

Stability analyses of parallel flows with slow timedependent or weakly non-parallel steady flows, from the mathematical point of view, are more complex than those of steady parallel flows, because the method of normal modes is not applicable. However, the squeeze flow varies slowly in space and time, so that the stability of such a flow is a little studied topic in the hydrodynamic-stability literature. Stuart et al. ${ }^{[3]}$ seem to be the first to have carried out the linear stability of the squeeze flow, with two parallel plates of small gaps moving normal to each other in a slow time-dependent speed, by using the WentzelKramers-Brillouin-Jeffries (WKBJ) technique. We will not refer to it here due to the complex theory fundamentals of WKBJ. Furthermore, when the weakly non-parallel basic flow is periodic in time, the Floquet theory can accurately predict the parametric instability of unsteady periodic flows, e.g. Hall and Papageorgiou $^{[4]}$ considered the flow in a squeeze bearing induced by the time-periodic oscillation of one of the walls.

Another investigation related to the present study is that of Aristov and Gitman, ${ }^{[5]}$ who considered the stability of the flow between two parallel discs moving normal to each other when the relative velocity $2 Q$ of the upper disc to the lower one is constant. In three-dimensional Cartesian coordinates $\left(x^{*}, y^{*}, z^{*}\right)$, the basic solution of the flow has the form $u_{x}^{*}=Q x^{*}$, $u_{y}^{*}=Q y^{*}$ and $u_{z}^{*}=-2 Q z^{*}$. The basic solution does

\footnotetext{
* Supported by the National Natural Science Foundation of China under Grant No 10472054, and Specialized Research Fund for the Doctoral Programme of Higher Education of China under No 20040003070.

** Email: zhukq@tsinghua.edu.cn

(C) 2005 Chinese Physical Society and IOP Publishing Ltd
} 
not satisfy the no-slip boundary conditions (BCs) and belongs to the inviscid type. The perturbed stream function $\Psi^{\prime}$ can be written in the form of a superposition of plane waves. The periodical one-dimensional disturbance is expressed by ${ }^{[5]}$

$$
\Psi^{\prime *}=k^{-2}\left(t^{*}\right) A\left(t^{*}\right) \cos \left[k\left(t^{*}\right) x^{*}\right],
$$

where the amplitude $A\left(t^{*}\right)$ and wavenumber $k\left(t^{*}\right)$ are dependent on time, and the disturbance is in the Kelvin-mode form, which is different from the common Tollmien-Schlichting wave. ${ }^{[6,7]}$

Aristov and Gitman ${ }^{[5]}$ only described the instability of the inviscid squeeze flow due to the limitation of Kelvin modes. In this Letter, we discuss whether the viscous basic flow is stable or not and how disturbances evolve. The instability analysis of the viscous squeeze film by using the Kelvin modes is not easy to proceed, because the treatment of the constraint of BCs possesses a difficulty. We do not address here the disturbance in the Kelvin modes, and the mode selection still deserves further studying in the future.

One ideally available and efficient tool for studying the global instability of weakly non-parallel flows (steady and unsteady periodic) is the low-dimensional Galerkin method (LDGM). The instability properties in the cylinder wake, the sphere wake, the boundarylayer and a variety of other flows are successfully carried out by employing an LDGM. ${ }^{[8-10]}$ This shows the method is valid for analysing the global instability of dynamic systems with slow time dependence and weakly non-parallel characters, such as the present problem.

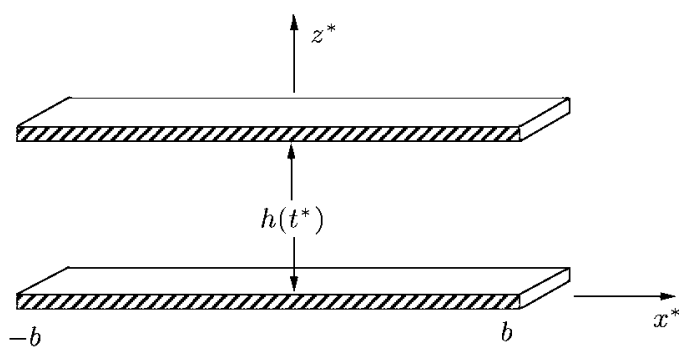

Fig. 1. Schematic of the planar squeeze flow.

We consider the two-dimensional motion of incompressible Newtonian fluids of kinematic viscosity $v$ between two parallel plates, the upper of which approaches the lower stationary one with a constant speed, as schematically depicted in Fig. 1. The breadth of plates is $2 b$; the time-dependent distance between the plates is $h\left(t^{*}\right)=2 a-U t^{*}$, where $2 a$ is the initial value of the distance, $t^{*}$ is time, and the relative velocity $U$ of the upper plate to the lower one is assumed to be constant for $U>0$ corresponding to the squeezing and $U<0$ to the dilatation. The dimensionless distance between the plates $H\left(n t^{*}\right)=(2 a)^{-1} h\left(t^{*}\right)$ has been introduced for mathematical convenience, where $H\left(n t^{*}\right)=1-\sigma n t^{*}$. The value of dimensionless speed of the upper plate relative to the lower one is $\sigma= \pm 1, \sigma>0$ for the squeezing and $\sigma<0$ for the dilatation. Thus $U=2 a \sigma n$ holds uniformly, where $n$ satisfies $1 / n=2 a / U$ and is the inverse of the characteristic time. The ratio of the distance between the plates at the initial point of time to their breadth is small, i.e. $\delta=a / b \ll 1$, and the Reynolds number is defined by $R e=2 a|U| / v$.

Following Stuart et al. ${ }^{[3]}$ we introduce dimensionless variables $t=n t^{*}, x=x^{*} b^{-1}, z=z^{*}\left[a H\left(n t^{*}\right)\right]^{-1}$ $1, u_{z}=u_{z}^{*}(n a)^{-1}, u_{x}=u_{x}^{*}(n b)^{-1} H\left(n t^{*}\right)$ and $\Psi=$ $\Psi^{*}(n a b)^{-1}$, where $-1 \leq x \leq 1$ and $-1 \leq z \leq 1$. For small values of $(4|\sigma|)^{-1} R e$, the basic solution $\Psi_{B}(z, x, t)$ takes the form ${ }^{[3]}$

$$
\Psi_{B}(z, x, t)=-x H^{\prime}(t)\left(2+3 z-z^{3}\right) / 2,
$$

where $H^{\prime}(t)=d H(t) / d t$. We suppose that the basic solution plus a perturbation can be written as $\Psi(z, x, t)=\Psi_{B}(z, x, t)+\Psi^{\prime}(z, x, t)$, where $\left|\Psi^{\prime}\right| \ll$ $\left|\Psi_{B}\right|$. The linearized differential equation satisfied by $\Psi^{\prime}$ is found to be

$$
\begin{aligned}
{[H(t) \partial / \partial t} & \left.-(z+1) H^{\prime}(t) \partial / \partial z\right] D^{2} \Psi^{\prime} \\
& -4|\sigma| H(t) D^{2} D^{2} \Psi^{\prime} / R e \\
& +\left(\partial \Psi_{B} / \partial z\right)\left(\partial D^{2} \Psi^{\prime} / \partial x\right) \\
& +\left(\partial D^{2} \Psi_{B} / \partial x\right)\left(\partial \Psi^{\prime} / \partial z\right) \\
& -\left(\partial \Psi_{B} / \partial x\right)\left(\partial D^{2} \Psi^{\prime} / \partial z\right) \\
& -\left(\partial D^{2} \Psi_{B} / \partial z\right)\left(\partial \Psi^{\prime} / \partial x\right),
\end{aligned}
$$

where the operator $D^{2}$ is given by

$$
D^{2}=\delta^{2} \partial^{2} / \partial x^{2}+\partial^{2} /\left[H^{2}(t) \partial z^{2}\right] .
$$

The homogeneous $\mathrm{BCs}$ are enforced by requiring

$$
\begin{aligned}
& \Psi_{z}^{\prime}\left|z=-1=0, \quad \Psi^{\prime}\right|_{z=-1}=0,\left.\quad \Psi_{z}^{\prime}\right|_{z=1}=0, \\
& \left.\Psi_{x}^{\prime}\right|_{z=1}=0 .
\end{aligned}
$$

We refer to the LDGM initiated by Noack and Eckelmann, ${ }^{[8,9]}$ and choose appropriate expansion modes. The disturbed stream function can be approximated by Fourier expansions with expansion modes, for which the Galerkin approximation reads

$$
\Psi^{\prime}(z, x, t)=\sum_{\substack{i=0,1, \cdots, k \\ j=0,1, \cdots, m}} a_{i j}(t) \Phi_{i}(x) Z_{j}(z),
$$

where $a_{i j}(t)$ denotes time-dependent Fourier coefficients, and $\Phi_{i}(x)$ and $Z_{j}(z)$ are the spanwise mode aligning with the breadth of the plates and the normal mode perpendicular to the plates, respectively. The essence of the LDGM demands that $\Phi_{i}(x)$ and $Z_{j}(z)$ of Eq. (6) should be orthonormal and satisfy the homogeneous BCs of Eq. (5).

There is some arbitrariness in the choice of the spanwise mode, which is unrestricted by the BCs and for instance can be chosen as trigonometric functions

$$
\begin{aligned}
& \Phi_{i}(x)=\cos (2 \mathrm{i} \pi x) \quad \text { or } \\
& \Phi_{i}(x)=\sin (2 i \pi x) \quad(i=0,1,2, \cdots, k),
\end{aligned}
$$


corresponding to the symmetric and antisymmetric modes, respectively and satisfying the orthonormality relationship $\left(\Phi_{i}, \Phi_{l}\right)=\int_{-1}^{1} \Phi_{i}(x) \Phi_{l}(x) d x=\delta_{i l}$, with the Kronecker symbol $\delta_{i l}=1$ for $i=l$ and $\delta_{i l}=0$ for the others.

The construction of the normal expansion mode is various if only the selected functions satisfy the orthonormality condition and homogeneous BCs $\left.Z_{j}(z)\right|_{z=-1}=0, \partial Z_{j}(z) /\left.\partial z\right|_{z=-1}=0,\left.Z_{j}(z)\right|_{z=1}=0$ and $\partial Z_{j}(z) /\left.\partial z\right|_{z=1}=0$. Here the normal mode is constructed as

$$
Z_{j}(z)=P_{j}(\xi) \times[1-\cos (2 \pi z)] \times[(2 j+1) / 3]^{1 / 2},
$$

where

$$
\xi=[\sin (4 \pi z)-8 \sin (2 \pi z)+12 \pi z] /(12 \pi) .
$$

with $-1 \leq \xi \leq 1$. $P_{j}(\xi)$ is a Legendre orthonormality polynomial of degree $j$ and $j=0,1,2, \cdots$, and satisfies the orthonormality relationship $\int_{-1}^{1} \frac{1}{2}(2 j+$ 1) $P_{j}(\xi) P_{n}(\xi) d \xi=\delta_{j n}$.

Substituting the Galerkin approximation (6) in the linearized disturbance equation (3), which is multiplied with the projection modes $\Phi_{p}(x) Z_{q}(z)$ and then integrated over the domain of the velocity field, we can obtain

$$
\begin{gathered}
\int_{-1}^{1} \int_{-1}^{1} R\left(a_{i j}\right) \Phi_{p}(x) Z_{q}(z) d x d z=0, \\
(i=0,1,2, \cdots, k ; j=0,1,2, \cdots, m) \\
(p=0,1,2, \cdots, k ; q=0,1,2, \cdots, m),
\end{gathered}
$$

where $R\left(a_{i j}\right)$ is the residuals of the disturbance equation. A linear system of first-order ordinary differential equations (ODEs) for the time-dependent Fourier coefficients (disturbance amplitudes), which has the modes number of $N=(k+1) \times(m+1)$ for symmetric spanwise modes and of $N=k \times(m+1)$ for antisymmetric ones, takes the form

$$
\boldsymbol{D}_{t} \boldsymbol{a}=\boldsymbol{A}(t) \boldsymbol{a},
$$

where $\boldsymbol{a}$ denotes $N$-vector $\left\{a_{l}\right\}^{T}$, the subscript $l$ is related to the subscript ij by $l=i \times(m+1)+(j+1)$ for symmetric spanwise modes and $l=(i-1) \times(m+$ $1)+(j+1)$ for antisymmetric ones. The $N \times N$ matrix $\boldsymbol{D}_{t}$, which is different for various values of $k$ and $m$, includes the differential operator $d / d t$. The $N \times N$ coefficient matrix $\boldsymbol{A}(t)$ is aperiodic in time.

Thus, for the given initial disturbance amplitudes $a_{i j}(0)$, solving the linearized disturbance equation is changed into an initial value problem of a linear system of first-order ODEs for the disturbance amplitudes. For steady or time-periodic parallel and weakly non-parallel basic flows, this system, which is autonomous or called a Floquet system, has many wellknown properties. For other unsteady weakly nonparallel basic flows discussed here, the explicit dependence of linear ODEs on time is aperiodic, so that the methods of normal modes and Floquet theory are not suitable. It is applicable to directly solve the initial value problem of linear ODEs.

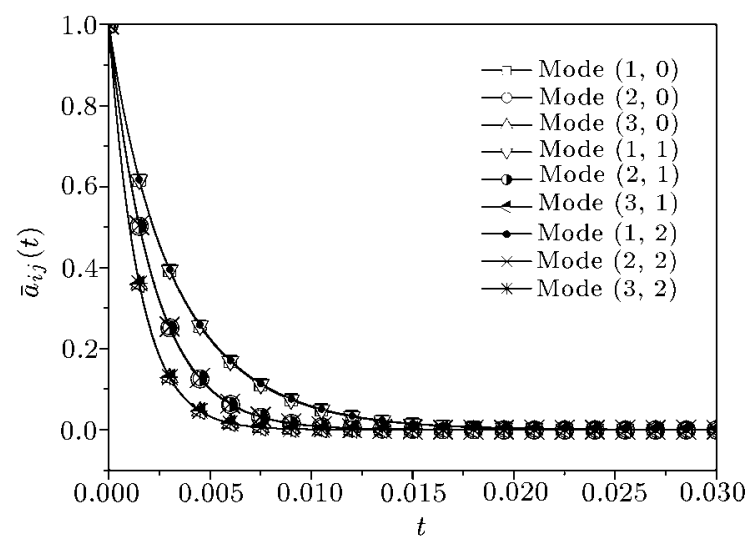

Fig. 2. Temporal evolution of disturbance amplitudes for squeezing.

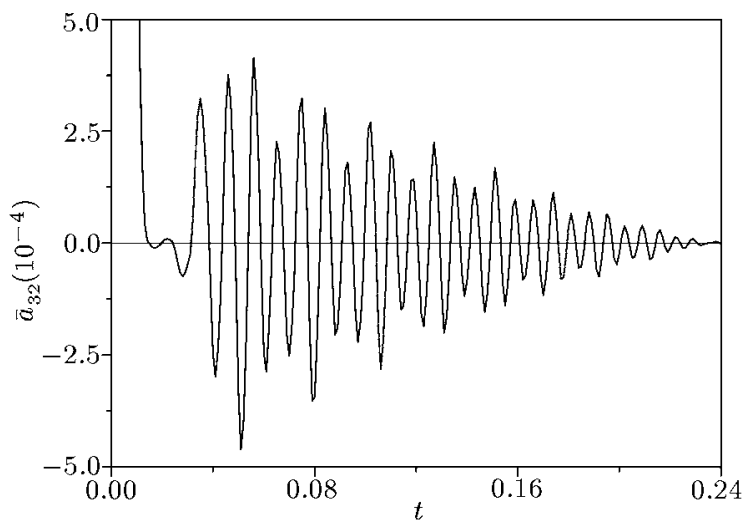

Fig. 3. One enlarged curve in Fig. 2 near the zero value of temporal evolution of the most unstable mode amplitude for squeezing.

In the calculation, we adopt the variables and values of some parameters as follows: antisymmetric spanwise disturbance modes, $\delta=0.01, a_{i j}(0)=$ $0.0001, k=3, m=2, R e=0.6$, unless specially stated. It is noted that the response of the basic flow to an infinitesimal disturbance of symmetric spanwise modes is similar to that of antisymmetric ones. Figures 2-4 show the dependences of the normalized disturbance amplitudes $\bar{a}_{i j}(t)=a_{i j}(t) / a_{i j}(0)$ on the dimensionless time, for different disturbance modes.

Aristov et $a ._{.}{ }^{[5]}$ suggested that when the plates are squeezed together, the inviscid solution is stable, with the amplitude decreasing in the course of time monotonically. Compared to the inviscid results, amplitudes of infinitesimal disturbances on the viscous laminar basic flow exhibit oscillatory decay with increasing time. The oscillatory amplitude values with respect to the initial disturbance become infinitesimal so that the oscillatory property in Fig. 2 is not easily observable. The amplitude in Fig. 2 near zero values 
of the most unstable mode is enlarged in Fig. 3, where the oscillatory decay of disturbance amplitude can be observed clearly. Furthermore, the tendency is evident in the initial time interval and the parameter $t$ of Fig. 2 only ranges from 0.0 to 0.03 because the amplitudes decay abruptly. Thus linear instability analyses show that the viscous squeezing motion is characterized by asymptotical stability.

From the physical mechanisms view of the instability, wall boundaries of the flow, which are an important factor affecting the flow instability, typically play the dual roles of stabilizer and destabilizer. On the one hand, they constrain the development of the disturbance and have stabilizing influences, the closer the plates being together the more stable the flow. On the other hand, they give rise to velocity shear that is diffused by viscosity and thereby tends to destabilize the flow. In the squeezing motion, the stabilizing role of boundaries is dominant and the diffusive effect of viscosity induces the oscillation near the zero value of disturbance amplitudes. Moreover, all the disturbance amplitudes should decay to zero when the plates are squeezed to some extent, i.e. the upper plate is very close to the lower one, because the disturbance exists in the flow field. This also verifies the reasonability and validity of the Galerkin method.

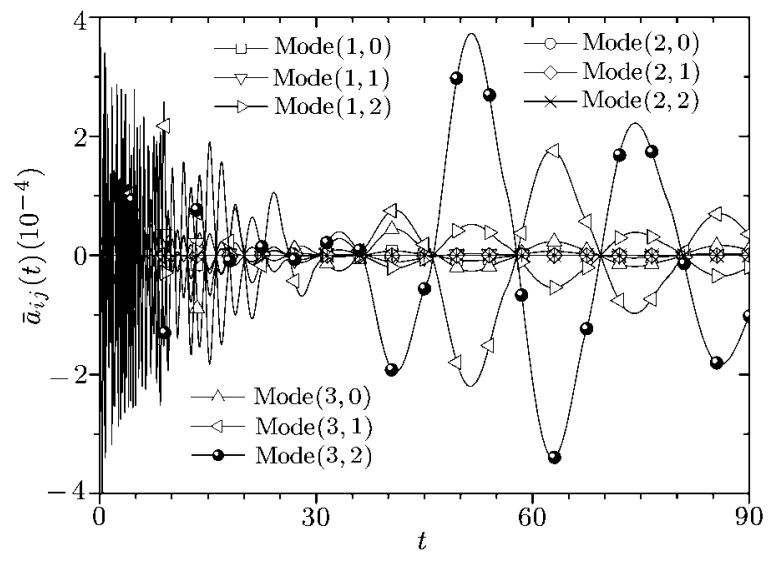

Fig. 4. Temporal evolution of disturbance amplitudes for dilatation.

When the plates are moving apart, the inviscid solution is asymptotically stable because the amplitude increases in the initial time interval but it will ultimately decay as time evolves. ${ }^{[5]}$ As is different from inviscid results, the evolution of the disturbance amplitudes in time exhibits oscillatory decay sharply and the viscous flow is asymptotically stable in the initial time interval. As the dilatation is continuously performed, the amplitudes exhibit oscillatory divergence, so that the flow will ultimately be unstable, as shown in Fig. 4, i.e. a closer view of the oscillation near the zero amplitude value.

From the physical view of instability, the constrained effect of wall boundaries in the dilatation mo- tion on the disturbance is stronger than the shear effect in the initial time interval due to low values of $R e$, and the flow tends to be asymptotically stable. With the increasing time and with the increasing gap between the plates, the constraint of the boundaries on the disturbance weakens, the lubrication assumption is no longer valid and the growth of disturbances strengthens in the viscous flow, so that the flow will ultimately be unstable.

The initial disturbance amplitudes should have no effect on the results under the condition of the linear equation and homogeneous BCs. As can be seen from the numerical computations, the tendency of the temporal evolution of amplitudes is identical for various initial disturbance amplitudes of the squeezing and dilatation motions. For example, the initial amplitude $a_{i j}(0)=0.005$ is ten times $a_{i j}(0)=0.0005$, correspondingly the amplitude curves of the former versus time are ten times larger than those of $a_{i j}(0)=0.0005$, which is consistent with the characteristics of the linear differential equation. As long as the initial amplitudes $a_{i j}(0)$ are small enough, the development of the disturbance is unrelated to $a_{i j}(0)$ and the nonlinear effects in the disturbance equation can be neglected. Therefore, temporal evolutions of amplitudes normalized by $a_{i j}(0)$ are the same as the curves in Figs. 2 and 4 , which further demonstrates a forcible validity of the scheme.

Though the temporal evolution of disturbances can reflect the global instability properties of a squeeze film basically, including the asymptotic behaviour of a long term or the transient behaviour of some time interval, there are some problems remained. On the one hand, the calculation will cost much with increasing numbers $N$ of disturbance modes. On the other hand, little is known about the stability of fluid flows with general time dependence, except for the autonomous and Floquet systems. Therefore, it is difficult to perform further investigations though the development of the disturbance amplitudes in time can be obtained. We apply the LDGM to analyse the global instability of viscous squeeze films and the results presented here require further experimental and numerical validations.

\section{References}

[1] Orazag S A 1971 J. Fluid Mech. 50689

[2] Hall P 1983 J. Fluid Mech. 126357

[3] Stuart J T et al 1990 Proc. R. Soc. London A 430347

[4] Hall P and Papageorgiou D T 1999 J. Fluid Mech. 39359

[5] Aristov S N and Gitman I M 2002 J. Fluid Mech. 464209

[6] Craik D D and Criminale W O 1986 Proc. R. Soc. London A 40613

[7] Kelvin L 1887 Philos. Magn. 24188

[8] Noack B R and Eckelmann H 1994 Phys. Fluids 6124

[9] Noack B R and Eckelmann H 1994 J. Fluid Mech. 270297

[10] Hu G H et al 1996 Phys. Fluids 8197 